\title{
Near-Infrared-Excitable Organic Ultralong Phosphorescence through Multiphoton Absorption
}

\author{
Ye Tao, ${ }^{1}$ Lele Tang, ${ }^{1}$ Qi Wei ${ }^{\circ},{ }^{2}$ Jibiao Jin, ${ }^{1}$ Wenbo Hu, ${ }^{1}$ Runfeng Chen, ${ }^{1}$ Qingqing Yang, \\ Huanhuan Li, ${ }^{1}$ Ping Li, ${ }^{1}$ Guichuan Xing, ${ }^{2}$ Quli Fan, ${ }^{1}$ Chao Zheng, ${ }^{1}$ and Wei Huang ${ }^{1,3}$ \\ ${ }^{1}$ Key Laboratory for Organic Electronics and Information Displays \& Jiangsu Key Laboratory for Biosensors, Institute of Advanced \\ Materials (IAM), Nanjing University of Posts \& Telecommunications, 9 Wenyuan Road, Nanjing 210023, China \\ ${ }^{2}$ Joint Key Laboratory of the Ministry of Education, Institute of Applied Physics and Materials Engineering, University of Macau, \\ Avenida da Universidade, Taipa, Macau 999078, China \\ ${ }^{3}$ Frontiers Science Center for Flexible Electronics (FSCFE), MIIT Key Laboratory of Flexible Electronics (KLoFE), Shaanxi Key \\ Laboratory of Flexible Electronics, Xi'an Key Laboratory of Flexible Electronics, Xi'an Key Laboratory of Biomedical Materials \\ \& Engineering, Xi'an Institute of Flexible Electronics, Institute of Flexible Electronics (IFE), Northwestern Polytechnical University, \\ Xi'an, 710072 Shaanxi, China
}

Correspondence should be addressed to Runfeng Chen; iamrfchen@njupt.edu.cn, Quli Fan; iamqlfan@njupt.edu.cn, and Wei Huang; iamdirector@fudan.edu.cn

Received 9 August 2020; Accepted 29 September 2020; Published 1 December 2020

Copyright (C) 2020 Ye Tao et al. Exclusive Licensee Science and Technology Review Publishing House. Distributed under a Creative Commons Attribution License (CC BY 4.0).

\begin{abstract}
Organic ultralong room-temperature phosphorescence (OURTP) with a long-lived triplet excited state up to several seconds has triggered widespread research interests, but most OURTP materials are excited by only ultraviolet (UV) or blue light owing to their unique stabilized triplet- and solid-state emission feature. Here, we demonstrate that near-infrared- (NIR-) excitable OURTP molecules can be rationally designed by implanting intra/intermolecular charge transfer (CT) characteristics into $\mathrm{H}$ aggregation to stimulate the efficient nonlinear multiphoton absorption (MPA). The resultant upconverted MPA-OURTP show ultralong lifetimes over $0.42 \mathrm{~s}$ and a phosphorescence quantum yield of $\sim 37 \%$ under both UV and NIR light irradiation. Empowered by the extraordinary MPA-OURTP, novel applications including two-photon bioimaging, visual laser power detection and excitation, and lifetime multiplexing encryption devices were successfully realized. These discoveries illustrate not only a delicate design map for the construction of NIR-excitable OURTP materials but also insightful guidance for exploring OURTP-based nonlinear optoelectronic properties and applications.
\end{abstract}

\section{Introduction}

Long-lived organic phosphorescence with lifetime over $0.1 \mathrm{~s}$ has shown great significance in both scientific understandings and technological applications ranging from anticounterfeiting [1-3], persistent light-emitting diodes [4], bioimaging $[5,6]$, and temperature sensing [7] to logic computing [8]. With various molecular design strategies including host-guest doping [9-12], $\mathrm{H}$-aggregation [1, 13-15], crystallization [16-18], polymerization [19-21], and metalorganic framework coordination [22], a large number of organic ultralong room-temperature phosphorescence (OURTP) materials have been developed with lifetimes up to $\sim 22.4 \mathrm{~s}$ and quantum efficiency over $40 \%$ under ambient conditions [7, 23]. However, compared to the abundant and vivid emission behaviors of OURTP showing blue, green, red, and white and even dynamically varied colors, most OURTP can only be excited by ultraviolet (UV) or blue light [24-26], owing to the intrinsic multiple exciton transformation features of OURTP (Figure 1(a)), where the photoexcited excitons on the lowest singlet excited state $\left(\mathrm{S}_{1}\right)$ should be transformed to the triplet exciton through intersystem crossing (ISC) on the high-lying triplet excited state $\left(\mathrm{T}_{n}\right)$ firstly, followed by internal conversion (IC) and triplet stabilization processes to form the stabilized $\mathrm{T}_{n}\left(\mathrm{~T}_{n}{ }^{*}\right)$ [24]. Therefore, the radiative decay of the lowest $\mathrm{T}_{n}{ }^{*}\left(\mathrm{~T}_{1}{ }^{*}\right)$ for the OURTP emission is doomed to have very large Stokes shift $(\sim 150 \mathrm{~nm})$ and inherently require high-energy UV and/or 


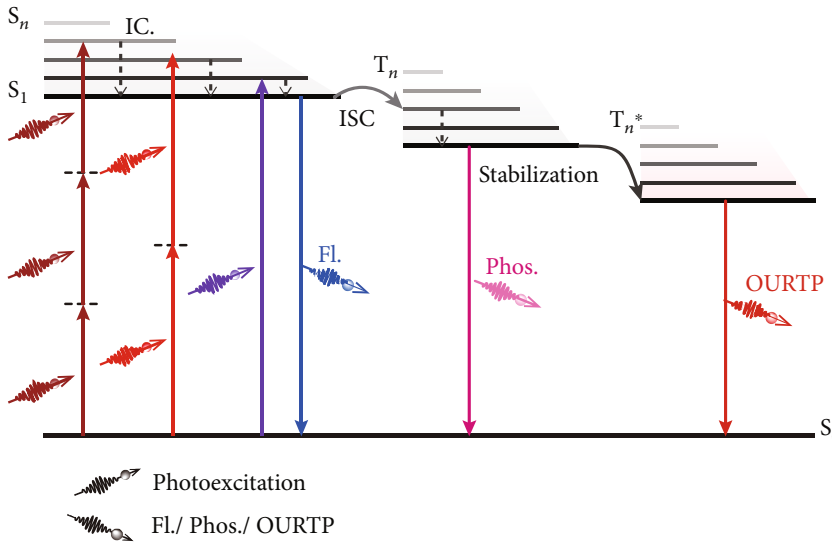

(a)

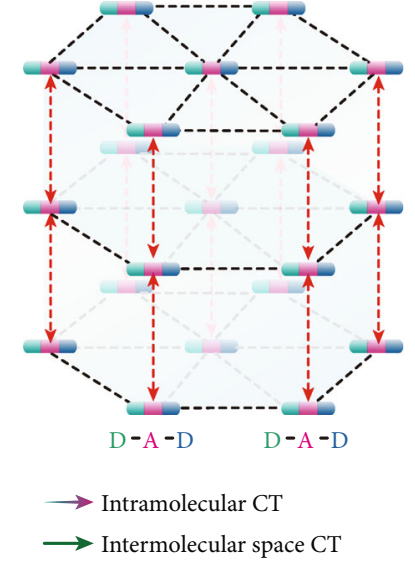

(b)

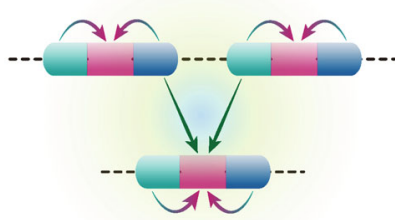

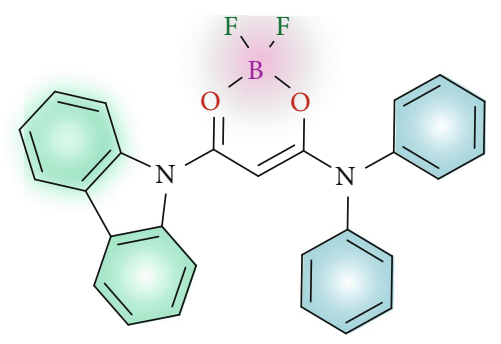

CzPAB

(c)

(d)

FIgURE 1: Molecular design strategy of MPA-OURTP materials. (a) Exciton transformation pathways of one-photon- (OPA), two-photon(TPA), and three-photon- (3PA) triggered OURTP. The ground state $\left(\mathrm{S}_{0}\right)$ molecule can be excited to $\mathrm{S}_{n}$ by absorption of one, two, or three photons and then fall to $\mathrm{S}_{1}$ through IC for fluorescence (Fl.). The triplet excited state $\left(\mathrm{T}_{n}\right)$ can be populated from $\mathrm{S}_{1}$ via ISC, and the radiative decay of the lowest $\mathrm{T}_{n}\left(\mathrm{~T}_{1}\right)$ leads to phosphorescence (Phos.), while by further stabilization for $\mathrm{T}_{n}{ }^{*}$, OURTP is produced. (b) Design of MPA-OURTP molecules in a D-A-D architecture with strong and abundant in-plane (dashed black) and interlayer (red line) intermolecular interactions in crystal. (c, d) Schematic drawing of (c) MPA-OURTP molecules using a planar $\pi$-conjugation donor and a difluoroboron $\beta$-diketonate acceptor with synergistic effects of intramolecular CT (ICT) and intermolecular space CT (SCT) for MPA and (d) the molecular structure of the designed model compound of CzPAB.

blue light excitation (Scheme S1). To reduce the Stokes shift and move the excitation wavelength to the visible range, rigid molecules with long conjugation lengths [27], direct triplet excited state absorption [14], and halogen/pseudohalogen atom incorporation have been proposed [28, 29], but the longest excitation wavelength is still shorter than $500 \mathrm{~nm}$. Considering the phototoxicity of UV and blue light, it is urgent to explore the low-energy light-excitable OURTP.

Multiphoton absorption (MPA) is a nonlinear process in which a molecule can be excited from its ground state $\left(\mathrm{S}_{0}\right)$ to the excited state by simultaneously absorbing two or more photons [30]. Therefore, the excitation wavelength can be significantly redshifted to even the near-infrared (NIR) range, if MPA is activated at large MPA cross section for the upconverted emission. Considerable success has been achieved in designing MPA-featured organic fluorophores $[31,32]$, phosphors [33], and thermally activated delayed fluorescence materials [34] in donor- $\pi$-acceptor (D- $\pi$-A) architectures with extended charge delocalization. However, it is notoriously challenging to develop the MPA-OURTP, owing to the intrinsic difficulties in simultaneously satisfying (i) the incorporation of strong charge transfer (CT) into an organic skeleton to enhance molecular dipole and conjugation for guaranteeing the large MPA cross section and highly efficient nonlinear optical behaviors [30, 35], (ii) the involvement of heteroatoms to confer efficient $n-\pi^{*}$ transition for boosting ISC [1], and (iii) the formation of definitely ordered molecular aggregation in a solid state for stabilizing the triplet excitons and suppressing the nonradiative decays [24].

Here, we propose a rational design strategy by embedding intra- and intermolecular CT characters into $\mathrm{H}$-aggregation in a quadrupolar D-A-D architecture for MPA-OURTP. Specifically, the synergistic effects of strong intramolecular CT (ICT) and intermolecular space CT (SCT) will increase MPA cross section for the upconverted emission, and the $\mathrm{H}$-aggregation will stabilize the triplet excitons for OURTP. With this strategy, we choose the strong electron-withdrawing difluoroboron $\beta$-diketonate $\left(\mathrm{BF}_{2} \mathrm{bdk}\right)$ 
as the central acceptor moiety and two $\pi$-conjugation arylamines of carbazole and diamine as donor units. This design can not only motivate efficient ICT and SCT between arylamines and $\mathrm{BF}_{2} \mathrm{bdk}$ to boost MPA in both single molecular and aggregated states $[36,37]$ but also facilitate ISC by the inherent nonbonding $p$ electrons of boron (B) and lone pair electrons of nitrogen $(\mathrm{N})$ and fluorine $(\mathrm{F})[24,38]$; meanwhile, various interlocked interactions empowered by the multiple heteroatom incorporation (Figures 1(b) and 1(c)) also result in the greatly suppressed nonradiative decay of the excited states for high luminescent efficiency; the implanted planar $\pi$-conjugation arylamine endows the construction of $\mathrm{H}$-aggregation, which is crucial in the stabilization of triplet excitons for OURTP.

\section{Results}

2.1. Synthesis and Photoluminescence Properties. As a proof of concept, difluoroboron 3-(9H-carbazol-9-yl)-3-oxo-N,Ndiphenylpropanamide (CzPAB) was synthesized and systematically characterized (Figures S1-S9). Indeed, intense blue steady-state photoluminescence (SSPL) and yellowish OURTP emission from the CzPAB powder under ambient conditions can be excited by not only $365 \mathrm{~nm}$ UV light (Figure 2(a), top panel) but also NIR laser at $720 \mathrm{~nm}$ (Figure 2(a), middle panel) and $800 \mathrm{~nm}$ (Figure 2(a), bottom panel). Interestingly, the SSPL and OURTP spectra excited at 365, 720, and $800 \mathrm{~nm}$ are almost identical, exhibiting the main fluorescence band at $430 \mathrm{~nm}$ with nanosecond lifetime (Figure S10) and OURTP band at $530 \mathrm{~nm}$ with lifetime around $0.4 \mathrm{~s}$ (Figure S11 and Table S1). From the excitation-phosphorescence mapping, the OURTP of CzPAB can be excited from 240 to $420 \mathrm{~nm}$ (Figure 2(b) and Figure S12) with quite a low incident light intensity (20\% Iris) and short irradiation duration time (< $0.1 \mathrm{~s}$ ) (Figure S13) as well as from the flashlight of a commercial mobile phone. The phosphorescence quantum yield of $\mathrm{CzPAB}$ powder at $365 \mathrm{~nm}$ excitation is as high as $37 \%$ under ambient conditions, which is among the best efficiencies of OURTP reported to date. To stimulate the nonlinear MPA process, 720 and $800 \mathrm{~nm}$ NIR lasers should be adopted and the higher laser power leads to stronger emissions (Figure 2(c)). From the power-dependent SSPL analyses, the integrated emission intensities are quadratic (slope $\sim 2.0$, Figure 2(c), inset top) and cubic (slope $\sim 3.3$, Figure 2(c), inset bottom) in response to the incident laser power, obviously verifying the two- and three-photonexcited luminescent feature at room temperature $[30,33]$. Importantly, the MPA-OURTP exhibits nearly the same ultralong lifetime around $400 \mathrm{~ms}$ as the one-photon-excited OURTP by UV light (Figures 2(d) and 2(e) and Figure S11), suggesting the same decay process of the excitons photoexcited either linearly or nonlinearly.

2.2. Theoretical Investigations. To gain deep insights into the extraordinary MPA-OURTP feature of $\mathrm{CzPAB}$ powder, density functional theory (DFT) and time-dependent DFT (TD-DFT) calculations were performed to investigate the electronic structures of the excited states on both the single molecular (SM) and aggregated dimer states. Natural transition orbital (NTO) analyses show the separation of the highest occupied NTO (HONTO) and the lowest unoccupied NTO (LUNTO) isosurface at both $S_{1}$ and $T_{1}$ states with small frontier orbital overlap integrals $\left(I_{\mathrm{S}}\right.$ and $\left.I_{\mathrm{T}}<28 \%\right)$ because of the strong ICT feature of CzPAB in the SM state (Figure 3(a)) [39]. Also, an apparent ICT character was observed at $S_{0}$ in the SM state with large CT amount $(q)$ over 0.7 and became more obvious in the aggregated dimer structures with $~ 1.4$ fold enhancement of $q$ [40] (Figure 3(b)) and more separated NTO distribution at $S_{1}$ with smaller $I_{S}$ with the aid of the additional SCT effect (Figure 3(a) and Figure S14). Extraordinarily, $I_{\mathrm{T}}$ of the dimer is significantly increased, which would be beneficial for the highly efficient phosphorescent emission. The theoretically predicted CT character was confirmed by both the broad structureless absorption band in dichloromethane (DCM) solution (Figure S15) and bathochromic shifted PL peaks at the increased solvent polarity (Figure S16). Again, owing to the CT nature, $\mathrm{CzPAB}$ exhibits a small singlet-triplet splitting energy $\left(\Delta E_{\mathrm{ST}}\right)$ of $0.29 \mathrm{eV}$ in solution and $0.18 \mathrm{eV}$ in powder estimated from the fluorescence and phosphorescence spectra at a cryogenic temperature of $77 \mathrm{~K}$ (Figure 3(c)). The reduced $\Delta E_{\mathrm{ST}}$ in powder should be due to the enhanced CT properties by the synergistic effects of ICT and SCT in the aggregated state. These small values of $\Delta E_{\mathrm{ST}}$ suggest that CzPAB should have facile ISC to populate $\mathrm{T}_{1}$ and reverse ISC (RISC) to return to $S_{1}$ for efficient thermally activated delayed fluorescence (TADF), which was experimentally observed on the OURTP spectra around $430 \mathrm{~nm}$ (Figure 2(a)) and theoretically confirmed by the Dolton simulations with the large spin orbital coupling (SOC) values between $S_{1}$ and $T_{n}$ (Figure 3(d) and Table S2) [7, 41]. The TADF-featured OURTP have greatly improved luminescent efficiency, since the spin-forbidden triplet state emission is transformed to the spin-allowed emission of the singlet excited state by the RISC process (Figure S17). Therefore, the OURTP quantum efficiency of CzPAB powder reaches $37 \%$, which is among the best results reported so far.

Besides the strong CT feature for the nonlinear MPA and small $\Delta E_{S T}$ for facile ISC to populate $\mathrm{T}_{1}, \mathrm{H}$-aggregations are also crucial in realizing the MPA-OURTP [42-44]. From Figure 3(e), many $\mathrm{H}$-aggregations with positive exciton splitting energies and strong $\pi-\pi$ interaction to stabilize the triplet excitons for OURTP emission were identified in $\mathrm{CzPAB}$ crystal by the Frenkel exciton theory (Table S4). Moreover, the central $\mathrm{CzPAB}$ is surrounded by six other molecules, exhibiting strong and abundant intermolecular interactions of $\mathrm{C}-\mathrm{H} \bullet \bullet \bullet \mathrm{C}, \mathrm{C}-\mathrm{B} \bullet \bullet \bullet \bullet \mathrm{H}, \mathrm{B}-\mathrm{F} \bullet \bullet \bullet \mathrm{H}$, and $\mathrm{C}-\mathrm{H} \bullet \bullet \bullet \bullet \mathrm{H}$ with corresponding distances of 2.891, 3.099, 2.597, and $2.355 \AA$, respectively (Figure $3(\mathrm{e})$, left). These strong intermolecular interactions can not only restrict the molecular vibration to suppress the nonradiative decays for the highly efficient emission but also provide solid evidence for the existence of SCT to reinforce the MPA ability in aggregated states.

2.3. Mechanism of MPA-OURTP. Based on these experimental and theoretical findings, a possible mechanism for MPAOURTP is proposed (Figure 4(a)). The D-A-D molecule with 

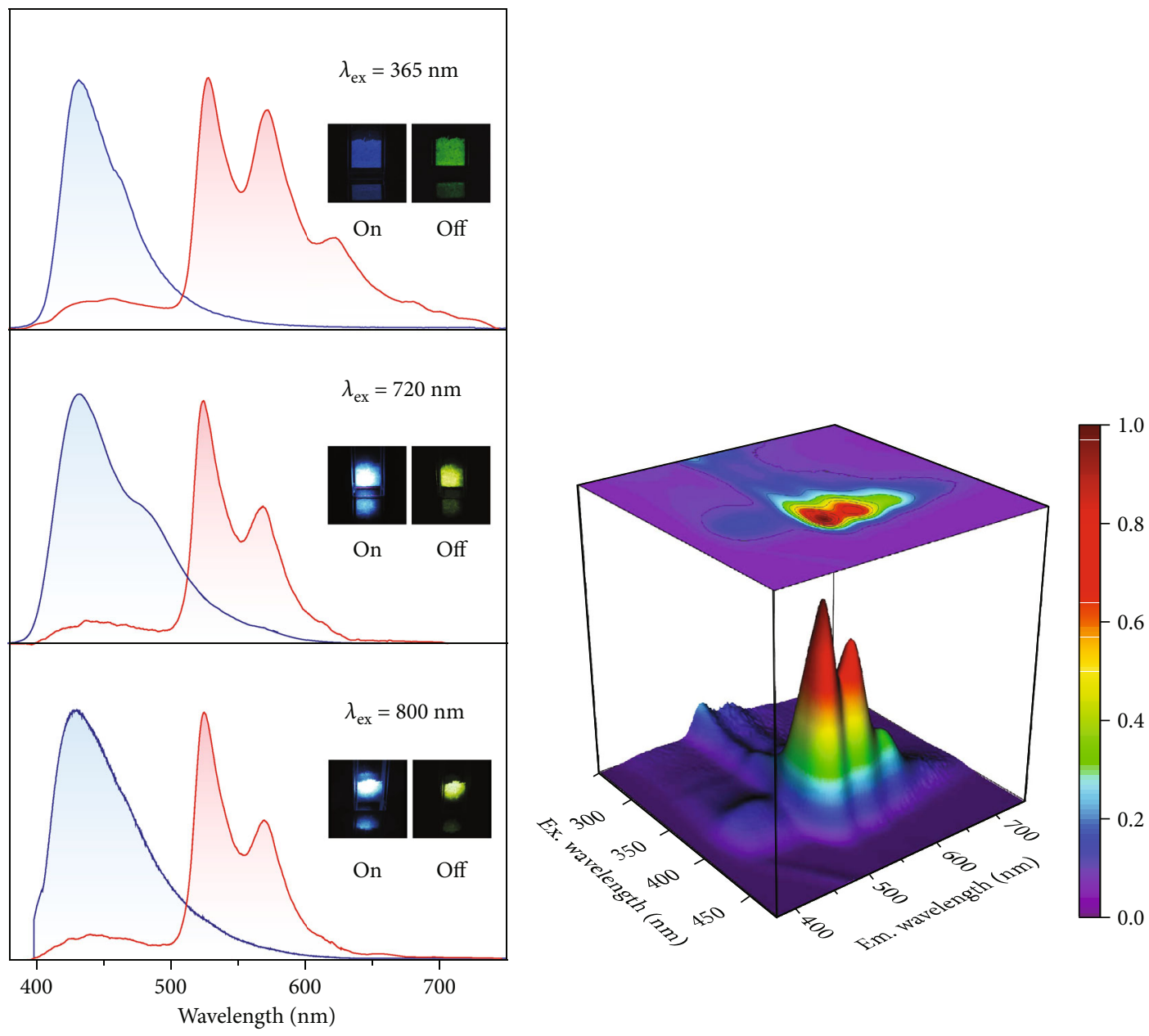

- PL

_ OURTP

(a)

(b)

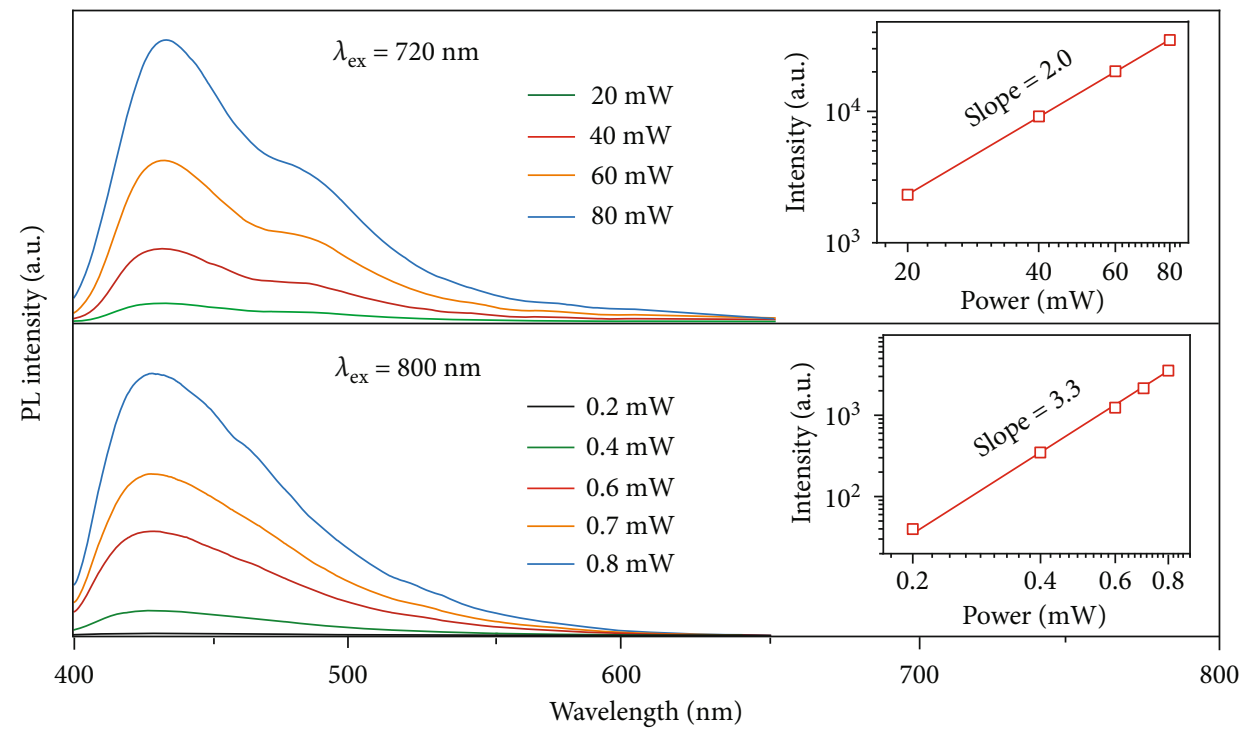

(c)

Figure 2: Continued. 


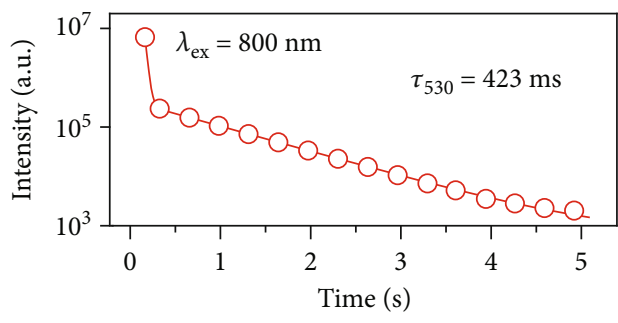

(d)

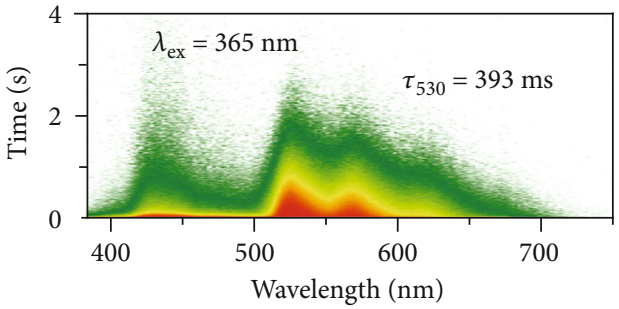

(e)

FIGURE 2: Photoluminescence properties of CzPAB powder under ambient conditions. (a) SSPL (blue) and OURTP (red) spectra excited by $365 \mathrm{~nm}$ UV light and 720 and $800 \mathrm{~nm}$ NIR lasers. Insets show the corresponding photographs on excitation (left) and removal (right) of the illumination light. (b) Excitation-OURTP emission mapping with a delay time of $25 \mathrm{~ms}$. (c) SSPL spectra under different strengths of 720 (top) and $800 \mathrm{~nm}$ (bottom) NIR lasers with logarithmic plots of the integrated emission intensity versus the laser powers (insets). (d) OURTP lifetime decay profile at $530 \mathrm{~nm}$ excited by an $800 \mathrm{~nm}$ laser. (e) Transient emission decay image excited by $365 \mathrm{~nm}$ UV light.

synergistic ICT and SCT effects in the solid state (Figure 4(b)) enables nonlinear MPA processes for NIR laser excitation to populate the singlet excited states, which transforms facilely to triplet ones by heteroatom facilitated ISC rates; the transformed triplet excitons are then stabilized by $\mathrm{H}$-aggregation and intermolecular interactions to slow down or suppress both the radiative and nonradiative decays (Figure 4(b)), resulting in the efficient MPA-OURTP from the radiative decay of the stabilized triplet excitons.

To confirm the above understandings in designing MPAOURTP molecules, we further prepared two $\mathrm{BF}_{2}$ bdk-based derivatives in a $\mathrm{D}-\mathrm{A}-\mathrm{D}$ molecular skeleton, namely, difluoroboron 1,3-di(9H-carbazol-9-yl) propane-1, 3-dione (DCzB) and difluoroboron $\mathrm{N}^{1}, \mathrm{~N}^{1}, \mathrm{~N}^{3}, \mathrm{~N}^{3}$-tetraphenylmalonamide (DPAB). Strong CT absorbance and emission peaks can be found in $\mathrm{DCzB}$ and DPAB solutions (Figures S18 and S19) due to the directly connected donor and acceptor units in the D-A-D architecture. Therefore, both DCzB and DPAB powders exhibit intense MPA-motivated fluorescence that is almost identical to their corresponding UV-triggered emission (Figures 4(c)-4(e)). For DCzB, obvious OURTP and MPA-OURTP were observed with lifetimes up to $\sim 230 \mathrm{~ms}$, when excited by both UV and NIR light (Figure 4(f), top panel). However, for DPAB, the phosphorescence lifetimes are only $\sim 58 \mathrm{~ms}$ (Figure 4(f), bottom panel, and Figure S20). To understand the different photophysical properties of $\mathrm{DCzB}$ and $\mathrm{DPAB}$, the single crystal structures of these two molecules were systematically investigated. Both $\mathrm{DCzB}$ and $\mathrm{DPAB}$ crystals display plenty of intermolecular interactions for the efficient SCT to facilitate the MPA process and suppress nonradiative decays, but compared to DCzB crystal with strong $\pi-\pi$ interaction (3.476 $\AA$ ) for $\mathrm{H}$-aggregations, DPAB exhibits loose $\pi-\pi$ interaction and J-aggregation dominates its solid state (Table S4). Therefore, no OURTP was observed in $\mathrm{DPAB}$ crystal, although its phosphorescence is quite strong with a high efficiency of $\sim 31 \%$. It is clear that efficient CT, facile ISC, $\mathrm{H}$-aggregation, and abundant intermolecular interactions are essential for efficient MPA-OURTP.

2.4. Applications of MPA-OURTP Materials. In light of the extraordinary linear and nonlinear photoexcitation feature of the MPA-OURTP materials, we tested the multifunctional applications of $\mathrm{CzPAB}$. Firstly, water-dispersible $\mathrm{CzPAB}$ nanoparticles with an average size of $\sim 90-100 \mathrm{~nm}$ and obvious fluorescence showing excellent photostabilities and OURTP emission with lifetime of $\sim 113 \mathrm{~ms}$ were prepared by the typical bottom-up approach using an amphiphilic copolymer (PEG-b-PPG-b-PEG, F127) [45] (Figures 5(a)5(c) and Figures S21 and S22). Two-photon confocal laser scanning microscopy images (Figure 5(d)) show that CzPAB nanoparticles can facilely permeate the cytoplasm of HeLa cells and emit strong MPA-OURTP with a high signal-to-noise ratio of $\sim 17$ by the $800 \mathrm{~nm}$ femtosecond laser irradiation, demonstrating the great potential of MPAOURTP materials in acquiring deep-tissue and highresolution bioimaging. Secondly, inspired by the MPAtriggered OURTP properties, we developed a novel visual NIR laser power detector (Figure 5(e)). From the OURTP photographs extracted from the video of $\mathrm{CzPAB}$ powder taken by a commercial mobile phone, the OURTP intensity is closely dependent on the laser powers (Figure 5(f) and Figure S23), and their $G$ values (Figure $5(\mathrm{~g})$ and Figure S24) are quantitatively related to the excitation laser power and laser off-time (Supporting Information, Sections S9 and S10). Therefore, the NIR laser powers can be obtained by correlating the $G$ value and laser off-time conveniently (Figure 5(g)). For instance, with the visual G values of 98, 103, and 108 at laser off-time of 1.5, 2.0, and $2.5 \mathrm{~s}$ located at 1, 2, and 3 in Figures 5(f) and 5(g), the excitation laser powers can be detected to be $0.2,0.3$, and $0.5 \mathrm{~W}$, respectively. Thirdly, we fabricated a new encryption device (Figure 5(h)) using DPAB; the UV, LED, and NIR light-excitable OURTP molecule of CzPAB; and other normal OURTP materials (Figure S25). With different excitation sources of handheld UV light, LED flashlight, and NIR laser, the lifetime-encrypted pattern varies from " 3 " and "7" to " 1 " (Figure 5(i)) correspondingly after switching off these excitation sources, demonstrating an interesting excitation and lifetime multiplexing feature of the anticounterfeiting device.

\section{Discussion}

In conclusion, we have proposed a rational molecular design strategy of upconverted OURTP materials to enable NIR- 

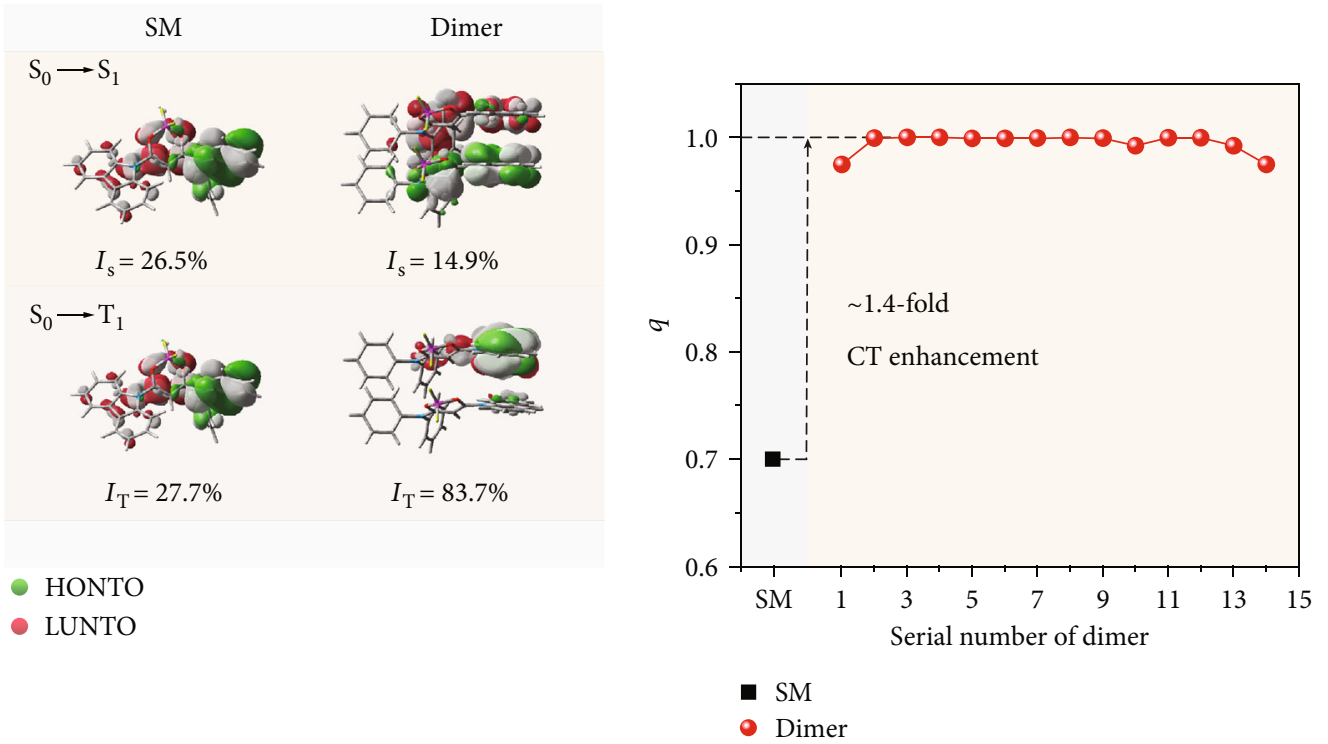

(a)
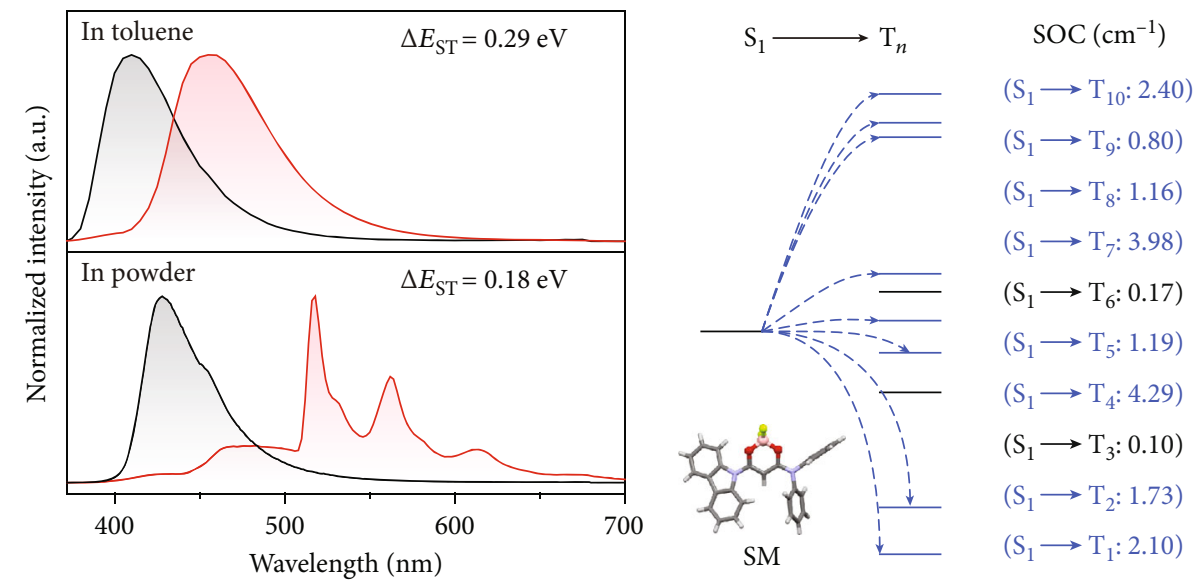

PL
Phos.

(c)

(d)

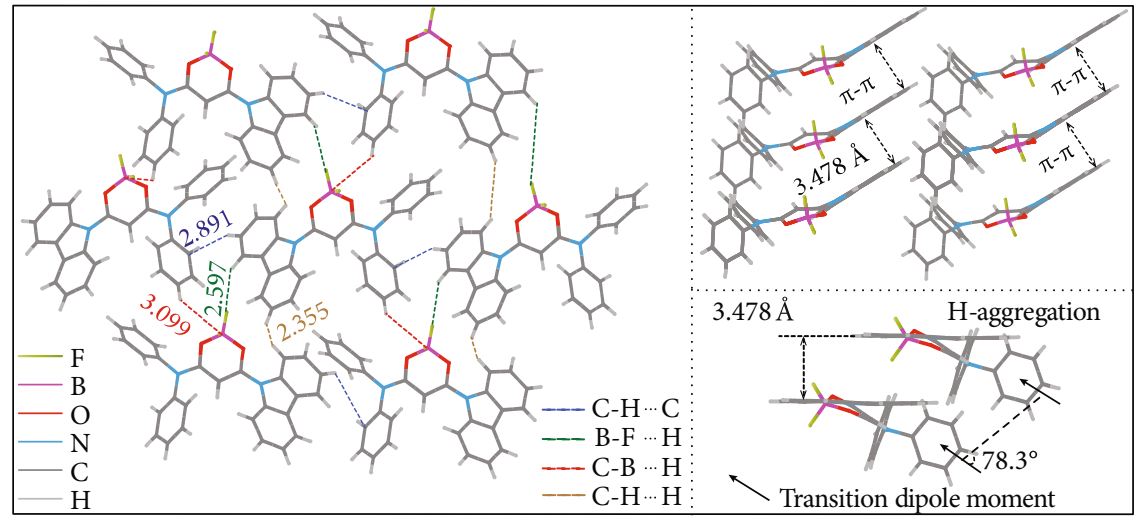

(e)

FIGURE 3: Theoretical and single-crystal analyses of CzPAB. (a) NTO analyses on $\mathrm{S}_{0} \rightarrow \mathrm{S}_{1}$ and $\mathrm{S}_{0} \rightarrow \mathrm{T}_{1}$ excitations and orbital overlap extents $\left(I_{\mathrm{S}}\right.$ and $I_{\mathrm{T}}$ ) at single molecular $(\mathrm{SM})$ and dimer states. (b) CT amount $(q)$ of SM and dimers extracted from the single crystal at the $\mathrm{S}_{0}$ state. (c) SSPL and phosphorescent (delay $5 \mathrm{~ms}$ ) spectra in toluene (top) and powder (bottom) at $77 \mathrm{~K}$. (d) TD-DFT-calculated excited state energy levels and the SOC constants between $\mathrm{S}_{1}$ and $\mathrm{T}_{n}$. (e) Molecular arrangement in single crystal with various intermolecular interactions (left) and representative molecular packing (top right) for $\mathrm{H}$-aggregation (bottom right). 


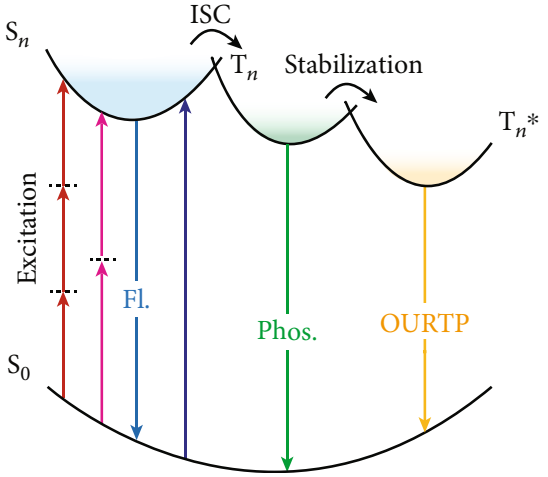

(a)

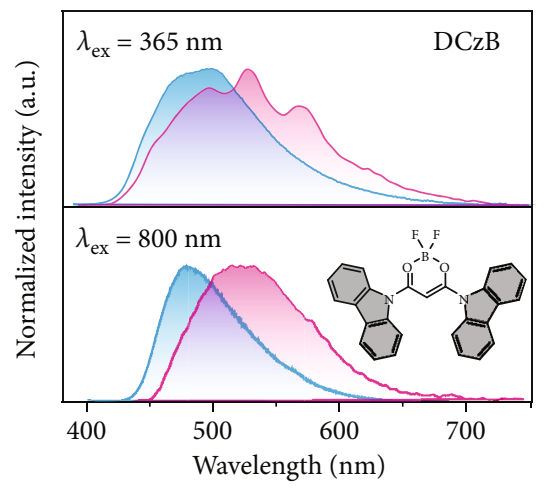

- PL

— OURTP

(c)

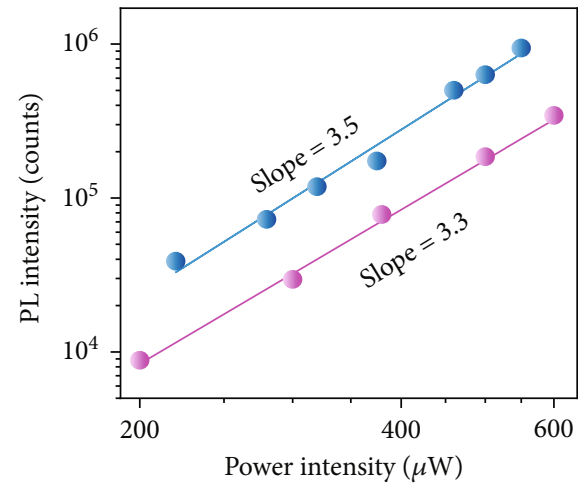

- DPAB

- $\mathrm{DCzB}$

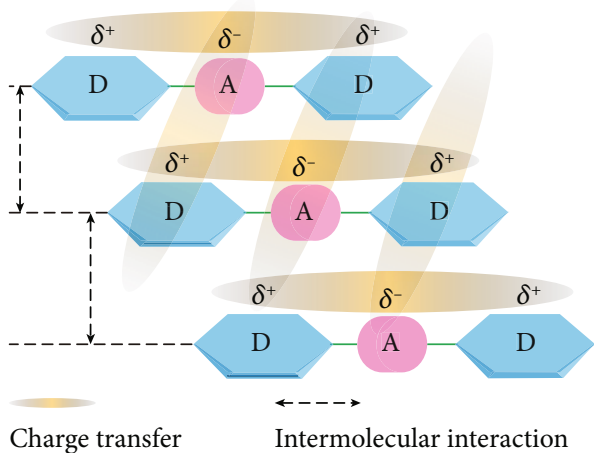

(b)

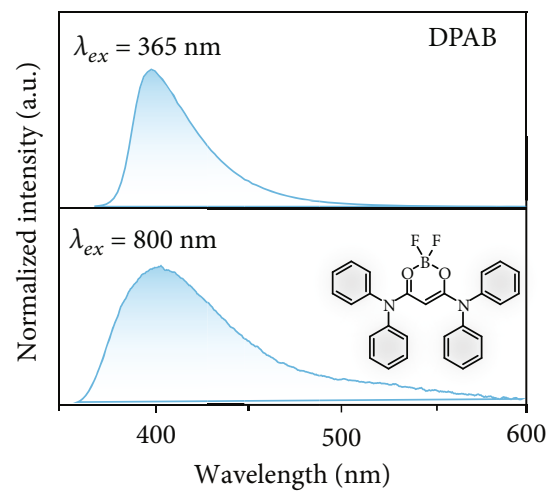

- PL

(d)

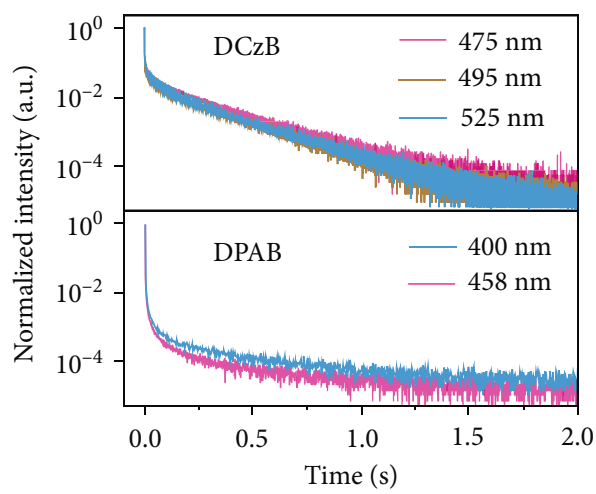

(f)

Figure 4: Continued. 


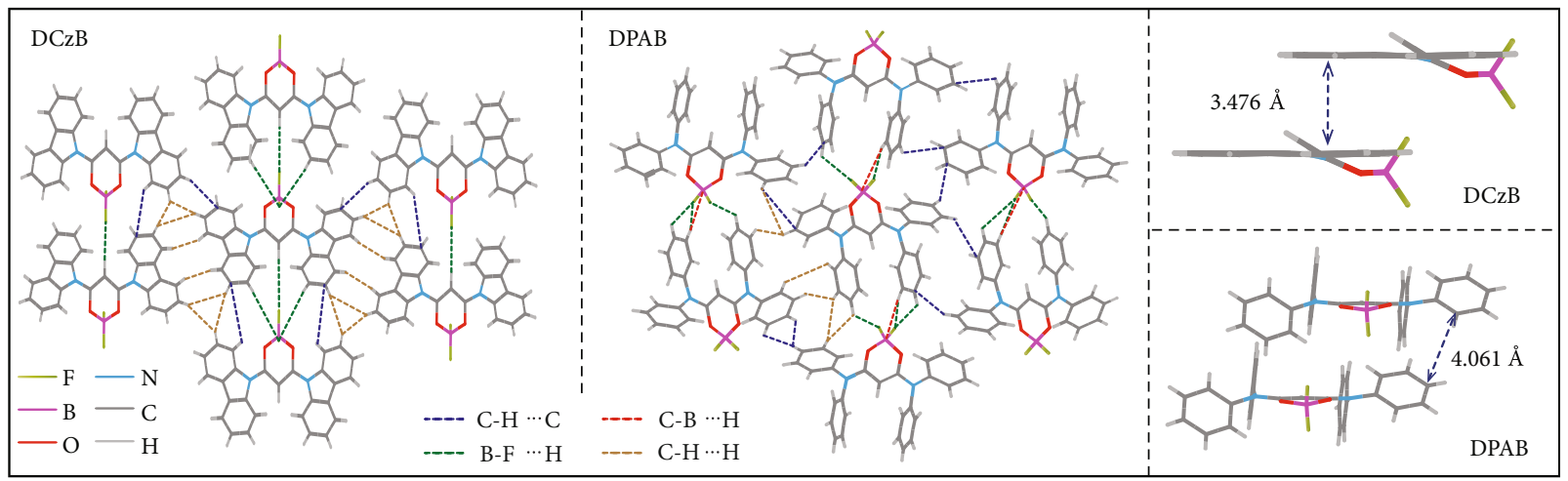

(g)

FIGURE 4: Proposed mechanism of MPA-OURTP and photophysical properties and aggregation structures of DCzB and DPAB powders. (a) Mechanisms of OURTP and MPA-OURTP. (b) Design principles of MPA-OURTP molecules by integrating intra- and intermolecular CT characters into H-aggregations in a quadrupolar D-A-D skeleton. (c, d) SSPL (blue) and OURTP (pink) spectra of the (c) DCzB and (d) DPAB powders excited by $365 \mathrm{~nm}$ UV light and $800 \mathrm{~nm}$ NIR laser. Insets show the corresponding molecular structures. (e) The logarithmic plots of the integrated emission intensity versus the $800 \mathrm{~nm}$ laser power. (f) OURTP lifetime decay profiles at 475 , 495 , and $525 \mathrm{~nm}$ for DCzB (top) and at 400 and $458 \mathrm{~nm}$ for DPAB (bottom) excited by $365 \mathrm{~nm}$ UV light. (g) Molecular arrangements in single crystals of $\mathrm{DCzB}$ (left) and DPAB (middle) with representative molecular packing structures.

excitable OURTP in small organic molecules with a quadrupolar D-A-D molecular skeleton. This strategy leans upon the insertion of ICT and SCT into $\mathrm{H}$-aggregation to render efficient MPA ability and stabilized triplet excitons in solid states for MPA-OURTP emission. The OURTP quantum yields and lifetimes reach up to $37 \%$ and $423 \mathrm{~ms}$, respectively. On account of the high-performance MPA-OURTP, twophoton-excited OURTP bioimaging, NIR laser power sensing, excitation and lifetime multiplexing encryption devices were applicable, illustrating a bright future of advanced applications with the nonlinear and NIR-excitable OURTP. We envision that the discovery of MPA-OURTP would stimulate intensive investigations on the nonlinear aspect of organic phosphors, providing a deep insight into the designing of rich upconverted photonic properties of OURTP for advanced and multifunctional device applications.

\section{Materials and Methods}

4.1. Preparation and Characterization of CZPAB. To a $50 \mathrm{~mL}$ double-neck bottle charged with $9 \mathrm{H}$-carbazole $(0.5 \mathrm{~g}$, $3.0 \mathrm{mmol})$ and diphenylamine $(0.5 \mathrm{~g}, 3.0 \mathrm{mmol})$ was injected $30 \mathrm{~mL}$ DCM using a syringe under an argon atmosphere. Then, the malonyl dichloride $(0.3 \mathrm{~mL}, 3.0 \mathrm{mmol})$ was injected to the reaction system slowly. After stirring at room temperature for 3 hours, a $\mathrm{BF}_{3} \cdot \mathrm{Et}_{2} \mathrm{O}\left(46.5 \% \mathrm{BF}_{3}, 1.2 \mathrm{~mL}, 9.0 \mathrm{mmol}\right)$ solution was added into the reaction mixture slowly. To complete the reaction, the mixture was refluxed overnight. The reaction mixture was quenched with $10 \mathrm{~mL}$ water and extracted with DCM for three times $(3 \times 100 \mathrm{~mL})$. The organic layers were collected, combined, and dried with anhydrous sodium sulfate $\left(\mathrm{Na}_{2} \mathrm{SO}_{4}\right)$. The solvent was removed under reduced pressure, and the residue was purified by column chromatography (silica gel, $3: 1 \mathrm{v} / \mathrm{v}$, petroleum ether/DCM). Yield: $0.35 \mathrm{~g}$ of white powder $(30 \%) .{ }^{1} \mathrm{H}$ NMR $(400 \mathrm{MHz}, \mathrm{d}-\mathrm{DMSO}, \mathrm{ppm}): \delta 8.22(\mathrm{~d}, J=7.5 \mathrm{~Hz}$, $2 \mathrm{H}), 7.86(\mathrm{~d}, J=7.7 \mathrm{~Hz}, 2 \mathrm{H}), 7.76(\mathrm{~d}, J=8.3 \mathrm{~Hz}, 2 \mathrm{H}), 7.71$ - $7.38(\mathrm{~m}, 12 \mathrm{H}), 5.34(\mathrm{~s}, 1 \mathrm{H}) .{ }^{13} \mathrm{C} \mathrm{NMR}\left(100 \mathrm{MHz}, \mathrm{CDCl}_{3}\right) \delta$ $169.3,164.7,138.0,130.4,129.5,128.0,127.1,126.4,126.3$, 123.7, 120.1, 114.8, 77.6. HRMS (ESI): $\mathrm{m} / \mathrm{z}$ calcd. for $\mathrm{C}_{27} \mathrm{H}_{20} \mathrm{BF}_{2} \mathrm{~N}_{2} \mathrm{O}_{2}[\mathrm{M}+\mathrm{H}]^{+}$, 453.1586; found, 453.1950.

4.2. Photophysical Measurements. Ultraviolet/visible (UV/Vis) absorption and photoluminescence (PL) spectra were recorded on a Jasco V-750 spectrophotometer and Edinburgh FLS980 spectrophotometer, respectively. The absolute photoluminescence quantum yield (PLQY) was obtained using an Edinburgh FLS980 fluorescence spectrophotometer equipped with an integrating sphere. For fluorescence decay measurements, a picosecond pulsed lightemitting diode (EPLED-380, wavelength: $377 \mathrm{~nm}$; pulse width: $947.7 \mathrm{ps)}$ was used. Phosphorescence spectra were obtained using an Edinburgh FLS980 fluorescence spectrophotometer at $77 \mathrm{~K}$ with a $10 \mathrm{~ms}$ delay time after excitation using a microsecond flash lamp. The microsecond flash lamp produces short, typically a few $\mu$ s, and high irradiance optical pulses for phosphorescence decay measurements in the range from microseconds to seconds. The kinetic measurements, OURTP spectra, and ultralong lifetime in powders were also measured on an Edinburgh FLS980 fluorescence spectrophotometer. For femtosecond optical spectroscopy, the laser source was a Coherent Legend regenerative amplifier $(150 \mathrm{fs}, 1 \mathrm{kHz}, 800 \mathrm{~nm})$ seeded with a Coherent Vitesse oscillator $(100 \mathrm{fs}, 80 \mathrm{MHz}) .800 \mathrm{~nm}$ wavelength laser pulses were from the regenerative amplifier's output. $720 \mathrm{~nm}$ laser pulses with pulse width $\sim 50$ fs were generated from an optical parametric amplifier (OperASolo) coupled to a one-box integrated Ti-Sapphire amplifier (Libra, Coherent). The emission from the samples was collected at a backscattering angle of $150^{\circ}$ by a pair of lenses and into an optical fiber that is coupled to a spectrometer (Acton, Spectra Pro 2500i) to be detected by a charge-coupled device (Princeton Instruments, Pixis 400B). The laser pulse (circular spot, diameter $1 \mathrm{~mm}$ ) is directly incident to the samples. 

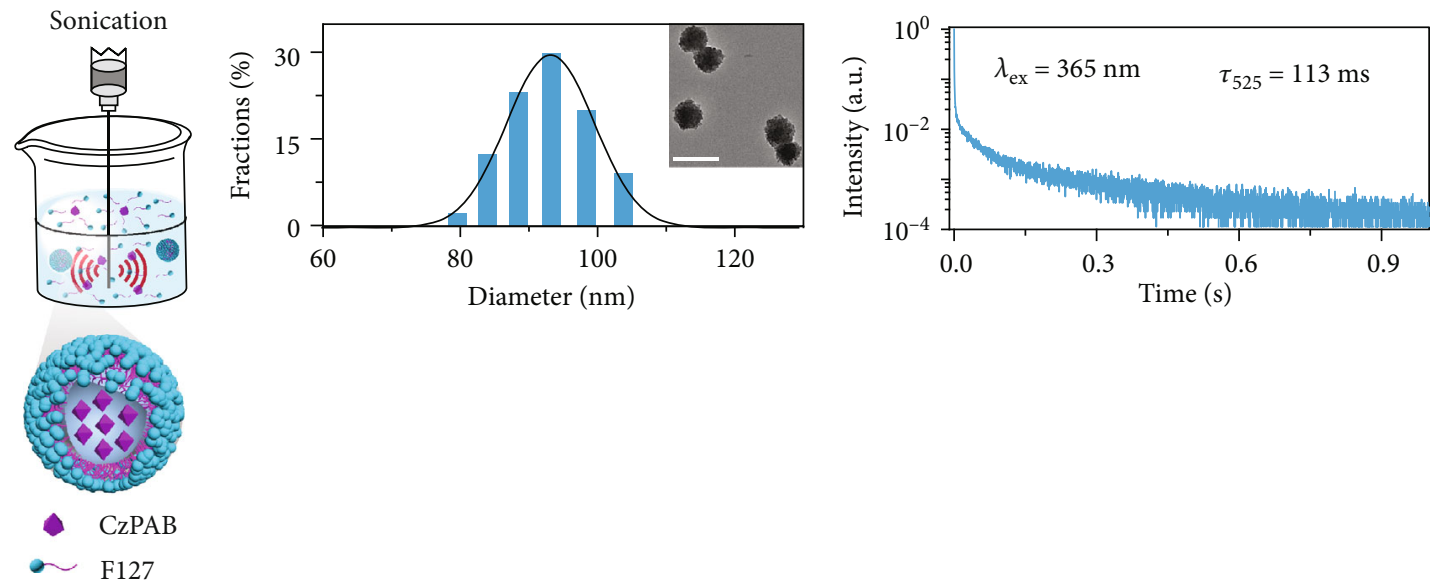

(a)

(b)

(c)

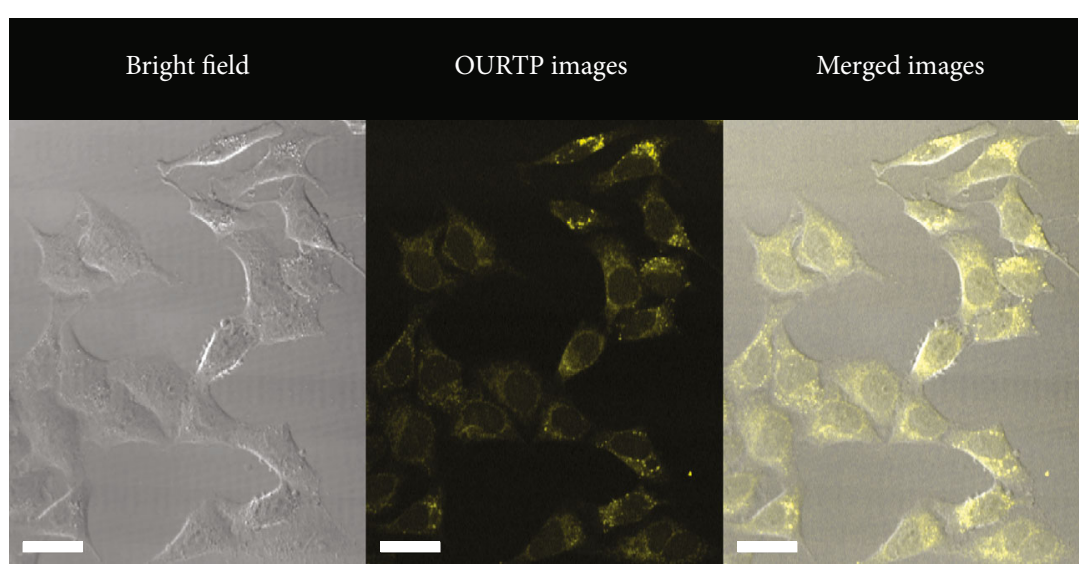

(d)

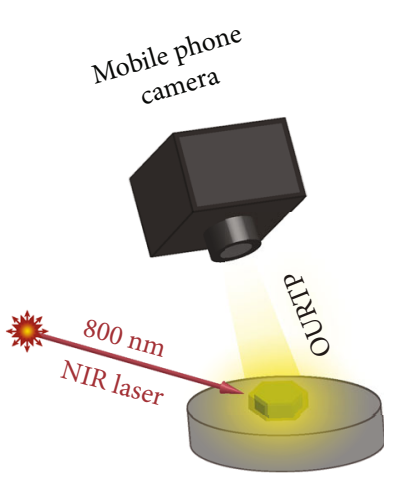

(e)

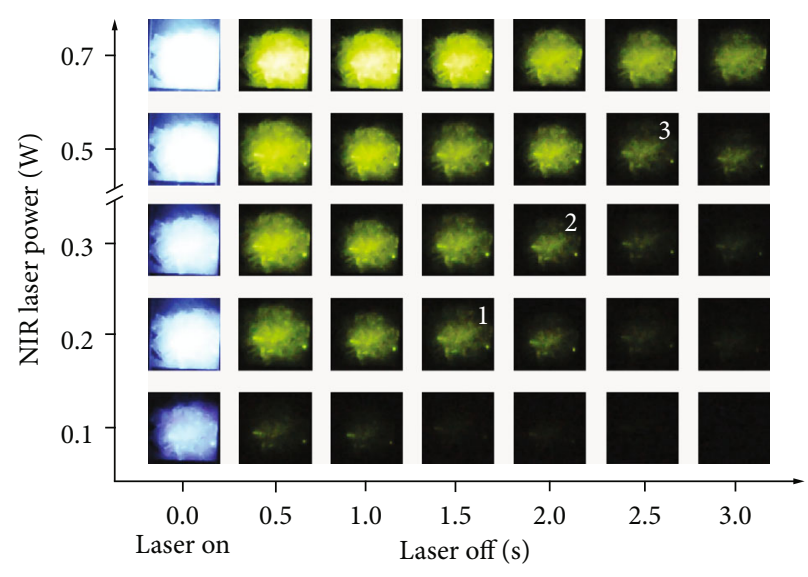

(f)

Figure 5: Continued. 


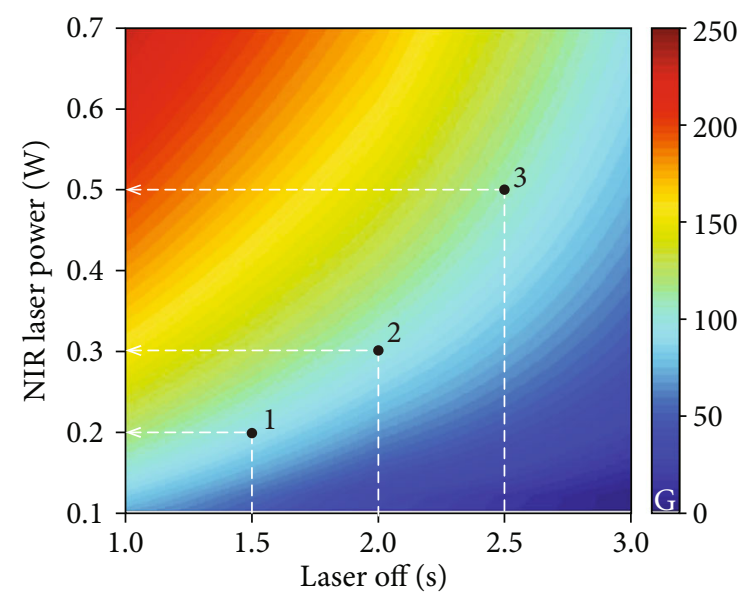

(g)

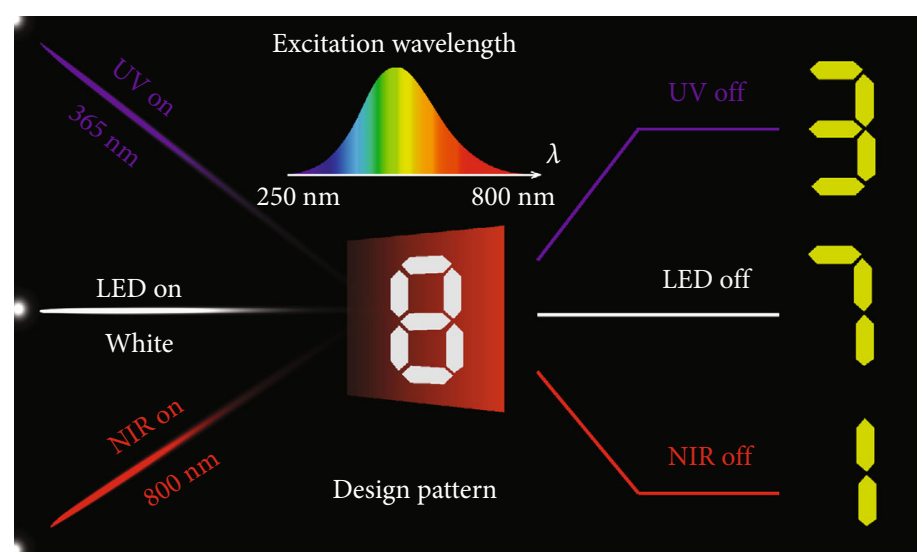

(h)

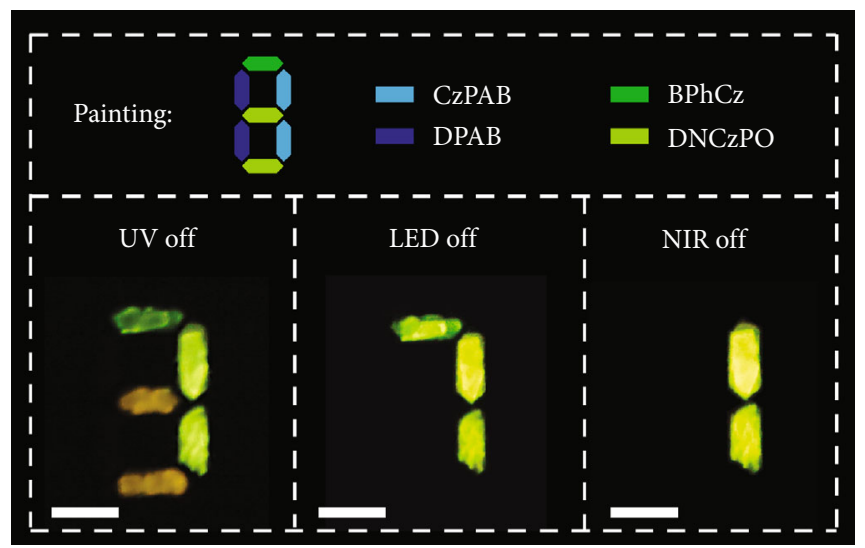

(i)

FIgURE 5: Applications of MPA-OURTP materials. (a) Schematic drawing of the bottom-up strategy to prepare CzPAB nanoparticles. (b) Particle size distribution revealed by dynamic light scattering. The inset is a transmission electron microscope image. The scale bar is $200 \mathrm{~nm}$. (c) OURTP lifetime decay profile at $530 \mathrm{~nm}$ of CzPAB nanoparticles excited by $365 \mathrm{~nm}$ UV light under ambient conditions. (d) Two-photon confocal laser scanning microscopy imaging of HeLa cells stained with CzPAB nanoparticles after incubation for $6 \mathrm{~h}$. The cellular images were captured by collecting the luminescence from 500 to $750 \mathrm{~nm}$ under the excitation of $800 \mathrm{~nm}$ NIR laser. The scale bar is $10 \mu \mathrm{m}$. (e) The setup for the visual NIR laser power detector. (f) Power-dependent OURTP images of CzPAB powder from the video recorded after turning off the $800 \mathrm{~nm}$ NIR laser. (g) Evolution mapping of grayscale $(\mathrm{G})$ values of the OURTP images under different laser off-time. (h) Design and (i) demonstration of the excitation and lifetime multiplexing encryption device. The scale bar is $1 \mathrm{~cm}$.

4.3. Theoretical Calculations. Density functional theory (DFT) and time-dependent DFT (TD-DFT) calculations were performed using the Gaussian 09 package. The ground-state geometries were optimized by the DFT method of the Lee-Yang-Parr correlation functional (B3LYP) using 6-31G(d) basis sets. The optimized static point was further carried out by harmonic vibration frequency analysis to guarantee that the real local minimum was achieved. The Dalton program with a quadratic response function method was used to predict spin-orbit coupling (SOC) matrix elements between the lowest singlet excited state $\left(\mathrm{S}_{1}\right)$ and the lowest triplet excited state $\left(\mathrm{T}_{1}\right)$. The SOC values were carried out on the basis of the optimized geometry of $\mathrm{T}_{1}$ using the B3LYP functional and 6-31G(d) basis set. Natural transition orbital (NTO) analysis was performed to get insights into the whole picture of the excited states with a compact orbital representation for the electronic transition density matrix. The overlap integrals between the highest occupied NTO
(HONTO) and the lowest unoccupied NTO (LUNTO) at $\mathrm{S}_{1}$ $\left(I_{\mathrm{S}}\right)$ and $\mathrm{T}_{1}\left(I_{\mathrm{T}}\right)$ states were also calculated using Multiwfn to take full considerations of electron transition components at the corresponding excited states.

For investigations on humans, a statement must be included indicating that informed consent was obtained after the nature and possible consequences of the study were explained.

\section{Conflicts of Interest}

The authors declare no competing financial interests.

\section{Authors' Contributions}

Y. Tao, L. Tang, R. Chen, and W. Huang conceived the experiments. L. Tang, J. Jin, H. Li, and C. Zheng performed the material synthesis, photophysical property characterization, 
and application fabrication. Q. Wei and G. Xing conducted the nonlinear optical measurements. W. Hu and Q. Fan carried out bioimaging experiments. Q. Yang and P. Li performed TD-DFT calculations. Y. Tao, L. Tang, R. Chen, and $\mathrm{W}$. Huang wrote the manuscript, with input from all the authors. Ye Tao, Lele Tang, and Qi Wei contributed equally to this work.

\section{Acknowledgments}

This work was financially supported in part by the National Natural Science Foundation of China (21772095, 21704042, 91833306, 61875090, 21674049, 61904152, and 21604039), the Six Talent Plan of Jiangsu Province (XCL-049), the 333 High-Level Talents Training Project of Jiangsu Province (BRA2019080), the Natural Science Fund for Colleges and Universities in Jiangsu Province (19KJA180005), the 1311 Talents Program of Nanjing University of Posts and Telecommunications (Dingfeng and Dingshan), the China Postdoctoral Science Foundation project (2018M642284), the Nanjing University of Posts and Telecommunications Start-up Fund (NUPTSF) (NY219007, NY217140, and NY219160), and the Science and Technology Innovation Project for Overseas Students in Nanjing.

\section{Supplementary Materials}

Scheme S1: a survey of representative single-component organic ultralong room-temperature phosphorescence molecules. Scheme S2: synthesis of MPA-OURTP molecules. Figure $\mathrm{S} 1:{ }^{1} \mathrm{H}$ NMR spectrum of $\mathrm{CzPAB}$ in d-DMSO. Figure S2: ${ }^{13} \mathrm{C}$ NMR spectrum of $\mathrm{CzPAB}$ in $\mathrm{CDCl} 3$. Figure S3: HRMS of CzPAB. Figure S4: ${ }^{13} \mathrm{H}$ NMR spectrum of DCzB in $\mathrm{CDCl} 3$. Figure S5: ${ }^{13} \mathrm{C}$ NMR spectrum of $\mathrm{DCzB}$ in $\mathrm{CDCl} 3$. Figure S6: HRMS of DCzB. Figure S7: ${ }^{1} \mathrm{H}$ NMR spectrum of DPAB in d-DMSO. Figure S8: ${ }^{13} \mathrm{C}$ NMR spectrum of DPAB in $\mathrm{CDCl} 3$. Figure S9: TGA curves of $\mathrm{CzPAB}, \mathrm{DCzB}$, and $\mathrm{DPAB}$. Figure S10: fluorescence decay profile of $\mathrm{CzPAB}$ powder at $300 \mathrm{~K}$. Figure S11: OURTP decay profiles of CzPAB powder at $300 \mathrm{~K}$. Figure S12: excitation spectrum of CzPAB by monitoring the emission peak at $530 \mathrm{~nm}$ and the luminescence spectrum of an iPhone 6's light-emitting diode (LED) light. Figure S13: photoluminescence intensity evolution under different excitation intensities and duration. Figure S14: molecular configuration of $\mathrm{CzPAB}$ dimers extracted from single crystals. Figure S15: photophysical properties in DCM solution. Figure S16: PL spectra of CzPAB in different solvents at room temperature. Figure S17: evolution of OURTP properties under different temperatures. Figure S18: absorption and fluorescence spectra of DCzB and DPAB in DCM solution. Figure S19: PL spectra of DCzB and DPAB in different solvents at room temperature. Figure S20: steady-state PL and phosphorescence spectra of DPAB crystal at $300 \mathrm{~K}$. Figure S21: PL properties of NPs. Figure S22: photostabilities of NPs. Figure S23: power-dependent OURTP images of $\mathrm{CzPAB}$ powder from the video recorded after turning off the $800 \mathrm{~nm}$ NIR laser. Figure S24: the G values versus incident laser power at different laser off-time. Figure S25: molecular structures and photophysical properties of
OURTP powder. Table S1: PL lifetimes of MPA-OURTP powders excited at $395 \mathrm{~nm}$ under ambient conditions Table S2: TD-DFT-calculated excited state energy levels and the spin-orbit coupling (SOC) constants between $\mathrm{S}_{1}$ and $\mathrm{T}_{n}$ of CzPAB. Table S3: crystallographic data of $\mathrm{CzPAB}, \mathrm{DCzB}$, and $\mathrm{DPAB}$. Table S4: aggregation structures in $\mathrm{CzPAB}$, $\mathrm{DCzB}$, and DPAB crystals identified by the exciton splitting energy $(\Delta \varepsilon)$. Movie S1: the CzPAB crystal excited by a 365 UV lamp, LED flashlight, and $800 \mathrm{~nm}$ NIR laser. Movie S2: the CzPAB crystal excited by $800 \mathrm{~nm}$ NIR laser under different excitations. (Supplementary Materials)

\section{References}

[1] Z. An, C. Zheng, Y. Tao et al., "Stabilizing triplet excited states for ultralong organic phosphorescence," Nature Materials, vol. 14, no. 7, pp. 685-690, 2015.

[2] L. Gu, H. Shi, L. Bian et al., "Colour-tunable ultra-long organic phosphorescence of a single-component molecular crystal," Nature Photonics, vol. 13, no. 6, pp. 406-411, 2019.

[3] C. C. Kenry and B. Liu, "Enhancing the performance of pure organic room-temperature phosphorescent luminophores," Nature Communications, vol. 10, no. 1, pp. 2111-2115, 2019.

[4] R. Kabe, N. Notsuka, K. Yoshida, and C. Adachi, "Afterglow organic light-emitting diode," Advanced Materials, vol. 28, no. 4, pp. 655-660, 2016.

[5] Q. Miao, C. Xie, X. Zhen et al., "Molecular afterglow imaging with bright, biodegradable polymer nanoparticles," Nature Biotechnology, vol. 35, no. 11, pp. 1102-1110, 2017.

[6] S. M. A. Fateminia, Z. Mao, S. Xu, Z. Yang, Z. Chi, and B. Liu, "Organic nanocrystals with bright red persistent roomtemperature phosphorescence for biological applications," Angewandte Chemie International Edition, vol. 56, no. 40, pp. 12160-12164, 2017.

[7] J. Jin, H. Jiang, Q. Yang et al., "Thermally activated triplet exciton release for highly efficient tri-mode organic afterglow," Nature Communications, vol. 11, no. 1, article 842, 2020.

[8] H. Li, H. Li, W. Wang et al., "Stimuli-responsive circularly polarized organic ultralong room temperature phosphorescence," Angewandte Chemie International Edition, vol. 59, no. 12 , pp. 4756-4762, 2020.

[9] R. Kabe and C. Adachi, "Organic long persistent luminescence,” Nature, vol. 550, no. 7676, pp. 384-387, 2017.

[10] S. Hirata, K. Totani, J. Zhang et al., "Efficient persistent room temperature phosphorescence in organic amorphous materials under ambient conditions," Advanced Functional Materials, vol. 23, no. 27, pp. 3386-3397, 2013.

[11] Y. Su, S. Z. F. Phua, Y. Li et al., "Ultralong room temperature phosphorescence from amorphous organic materials toward confidential information encryption and decryption," Science Advances, vol. 4, no. 5, article s9732, 2018.

[12] R. Gao, X. Mei, D. Yan, R. Liang, and M. Wei, "Nano-photosensitizer based on layered double hydroxide and isophthalic acid for singlet oxygenation and photodynamic therapy," Nature Communications, vol. 9, no. 1, p. 2798, 2018.

[13] X. Zhen, Y. Tao, Z. An et al., "Ultralong phosphorescence of water-soluble organic nanoparticles for in vivo afterglow imaging," Advanced Materials, vol. 29, no. 33, article 1606665, 2017.

[14] J. Yuan, R. Chen, X. Tang et al., "Direct population of triplet excited states through singlet-triplet transition for visible- 
light excitable organic afterglow," Chemical Science, vol. 10, no. 19, pp. 5031-5038, 2019.

[15] Y. Tao, R. Chen, H. Li et al., "Resonance-activated spinflipping for efficient organic ultralong room-temperature phosphorescence," Advanced Materials, vol. 30, no. 44, article 1803856, 2018.

[16] Y. Gong, G. Chen, Q. Peng et al., “Achieving persistent room temperature phosphorescence and remarkable mechanochromism from pure organic luminogens," Advanced Materials, vol. 27, no. 40, pp. 6195-6201, 2015.

[17] J. Yang, X. Zhen, B. Wang et al., "The influence of the molecular packing on the room temperature phosphorescence of purely organic luminogens," Nature Communications, vol. 9, no. 1, p. $840,2018$.

[18] W. Zhao, T. S. Cheung, N. Jiang et al., "Boosting the efficiency of organic persistent room-temperature phosphorescence by intramolecular triplet-triplet energy transfer," Nature Communications, vol. 10, no. 1, p. 1595, 2019.

[19] X. Ma, J. Wang, and H. Tian, “Assembling-induced emission: an efficient approach for amorphous metal-free organic emitting materials with room-temperature phosphorescence," Accounts of Chemical Research, vol. 52, no. 3, pp. 738-748, 2019.

[20] X. Ma, C. Xu, J. Wang, and H. Tian, "Amorphous pure organic polymers for heavy-atom-free efficient room-temperature phosphorescence emission," Angewandte Chemie International Edition, vol. 57, no. 34, pp. 10854-10858, 2018.

[21] S. Cai, H. Ma, H. Shi et al., "Enabling long-lived organic room temperature phosphorescence in polymers by subunit interlocking," Nature Communications, vol. 10, no. 1, p. 4247, 2019.

[22] X. Yang and D. Yan, "Long-afterglow metal-organic frameworks: reversible guest-induced phosphorescence tunability," Chemical Science, vol. 7, no. 7, pp. 4519-4526, 2016.

[23] S. Hirata and M. Vacha, "White afterglow room-temperature emission from an isolated single aromatic unit under ambient condition," Advanced Optical Materials, vol. 5, no. 5, article 1600996, 2017.

[24] S. Xu, R. Chen, C. Zheng, and W. Huang, "Excited state modulation for organic afterglow: materials and applications," Advanced Materials, vol. 28, no. 45, pp. 9920-9940, 2016.

[25] Z. He, W. Zhao, J. W. Y. Lam et al., "White light emission from a single organic molecule with dual phosphorescence at room temperature," Nature Communications, vol. 8, no. 1, p. 416, 2017.

[26] L. Gu, H. Wu, H. Ma et al., "Color-tunable ultralong organic room temperature phosphorescence from a multicomponent copolymer," Nature Communications, vol. 11, no. 1, p. 944, 2020.

[27] D. Tu, S. Cai, C. Fernandez et al., "Boron-cluster-enhanced ultralong organic phosphorescence," Angewandte Chemie International Edition, vol. 58, no. 27, pp. 9129-9133, 2019.

[28] S. Cai, H. Shi, J. Li et al., "Visible-light-excited ultralong organic phosphorescence by manipulating intermolecular interactions," Advanced Materials, vol. 29, no. 35, article 1701244, 2017.

[29] Y. Wang, Z. Zhang, L. Liu et al., "Cyanophenylcarbazole isomers exhibiting different UV and visible light excitable room temperature phosphorescence," Journal of Materials Chemistry C, vol. 7, no. 31, pp. 9671-9677, 2019.

[30] G. S. He, L. Tan, Q. Zheng, and P. N. Prasad, "Multiphoton absorbing materials: molecular designs, characterizations, and applications," Chemical Reviews, vol. 108, no. 4, pp. 1245-1330, 2008.

[31] Q. Zheng, H. Zhu, S. C. Chen, C. Tang, E. Ma, and X. Chen, "Frequency-upconverted stimulated emission by simultaneous five-photon absorption," Nature Photonics, vol. 7, no. 3, pp. 234-239, 2013.

[32] L. Sun, W. Zhu, W. Wang et al., "Intermolecular chargetransfer interactions facilitate two-photon absorption in styrylpyridine-tetracyanobenzene cocrystals," Angewandte Chemie International Edition, vol. 56, no. 27, pp. 7831-7835, 2017.

[33] R. Medishetty, J. K. Zaręba, D. Mayer, M. Samoć, and R. A. Fischer, "Nonlinear optical properties, upconversion and lasing in metal-organic frameworks," Chemical Society Reviews, vol. 46, no. 16, pp. 4976-5004, 2017.

[34] Y.-F. Xiao, J.-X. Chen, S. Li et al., "Manipulating exciton dynamics of thermally activated delayed fluorescence materials for tuning two-photon nanotheranostics," Chemical Science, vol. 11, no. 3, pp. 888-895, 2020.

[35] Z. Mao, Z. Yang, C. Xu et al., "Two-photon-excited ultralong organic room temperature phosphorescence by dual-channel triplet harvesting," Chemical Science, vol. 10, no. 31, pp. 7352-7357, 2019.

[36] S. K. Mellerup and S. Wang, "Boron-based stimuli responsive materials," Chemical Society Reviews, vol. 48, no. 13, pp. 3537-3549, 2019.

[37] X.-F. Wang, H. Xiao, P.-Z. Chen et al., "Pure organic room temperature phosphorescence from excited dimers in selfassembled nanoparticles under visible and near-infrared irradiation in water," Journal of the American Chemical Society, vol. 141, no. 12, pp. 5045-5050, 2019.

[38] W. Zhao, Z. He, J. W. Y. Lam et al., "Rational molecular design for achieving persistent and efficient pure organic roomtemperature phosphorescence," Chem, vol. 1, no. 4, pp. 592 602, 2016.

[39] T. Chen, L. Zheng, J. Yuan et al., "Understanding the control of singlet-triplet splitting for organic exciton manipulating: a combined theoretical and experimental approach," Scientific Reports, vol. 5, no. 1, article 10923, 2015.

[40] S. Huang, Q. Zhang, Y. Shiota et al., "Computational prediction for singlet-and triplet-transition energies of chargetransfer compounds," Journal of Chemical Theory and Computation, vol. 9, no. 9, pp. 3872-3877, 2013.

[41] H. Ma, Q. Peng, Z. An, W. Huang, and Z. Shuai, "Efficient and long-lived room-temperature organic phosphorescence: theoretical descriptors for molecular designs," Journal of the American Chemical Society, vol. 141, no. 2, pp. 1010-1015, 2019.

[42] D. Chaudhuri, D. Li, Y. Che et al., "Enhancing long-range exciton guiding in molecular nanowires by $\mathrm{H}$-aggregation lifetime engineering," Nano Letters, vol. 11, no. 2, pp. 488-492, 2011.

[43] F. Meinardi, M. Cerminara, A. Sassella, R. Bonifacio, and R. Tubino, "Superradiance in molecular H aggregates," Physical Review Letters, vol. 91, no. 24, article 247401, 2003.

[44] E. Lucenti, A. Forni, C. Botta et al., "H-aggregates granting crystallization-induced emissive behavior and ultralong phosphorescence from a pure organic molecule," The Journal of Physical Chemistry Letters, vol. 8, no. 8, pp. 1894-1898, 2017.

[45] Y. Jiang, J. Huang, X. Zhen et al., "A generic approach towards afterglow luminescent nanoparticles for ultrasensitive in vivo imaging," Nature Communications, vol. 10, no. 1, article 2064, 2019. 